УДК 616.12.89:616.125.2:616.089.819.1

DOI 10.17802/2306-1278-2018-7-3-6-11

\title{
ACHIEVING LOW RADIATION EXPOSURE AND CONTRAST USAGE WITHOUT COMPROMISING SAFETY AND EFFICACY DURING WATCHMAN LEFT ATRIAL APPENDAGE OCCLUSION DEVICE IMPLANT
}

\author{
J.A. Reiss ${ }^{1 \ltimes}$, D.A. Evans ${ }^{2}$
}

${ }^{1}$ PeaceHealth Southwest Medical Center, Heart \& Vascular, 200 NE Mother Joseph Place, Suite 400, Vancouver, WA 98664, United States; ' 2 PeaceHealth Southwest Medical Center, Performance Improvement, 200 NE Mother Joseph Place, Suite 400, Vancouver, WA 98664, United States;

\section{Highlights}

- The study demonstrates the safety, feasibility and effectiveness of a comprehensive approach to minimize fluoroscopy and contrast use during Watchman left atrial closure device implantation.

The introduction of Watchman left atrial appendage occlusion device (WM) has provided an effective alternative to anticoagulation for patients with a high risk of cerebrovascular accidents (CVA) and high risk of bleeding and who are unable

to take long-term anticoagulation therapy. Since its introduction, WM has been implanted more than 50,000 times worldwide. While the implant procedure is relatively safe, it involves the use of fluoroscopy and contrast and, as such, poses some associated risk to patient safety. The adoption of procedural techniques which reduce fluoroscopy exposure and contrast use have the potential to provide clinical patient benefit without compromising safety or efficacy.

$\begin{array}{ll}\text { Aim } & \begin{array}{l}\text { To demonstrate that WM implant can be performed with minimal exposure to both } \\ \text { ionizing radiation and IV contrast without compromising safety or efficacy. }\end{array}\end{array}$
A retrospective chart review of all 75 consecutive Watchman implantations by a single operator at a single center between December 2015 and December 2017. Every effort to optimize the WM implant procedure and minimize radiation and contrast exposure was incorporated as implant techniques evolved. Contrast and radiation exposure data were collected and analyzed year-over-year.

Charts from 75 consecutive cases were reviewed with all cases at index procedure $(100 \%)$, and included the majority of patients presenting in paroxysmal AF $(63 \%)$. Baseline patient characteristics were consistent across years. Procedural characteristics also were consistent over time. The median absorbed radiation dose

Results was low (75 mGy in 2015) and did not change significantly over time. Similarly, the median fluoroscopy time used after the initial case was low (2.8 minutes) and did not vary. 73 of $75(97 \%)$ of procedures resulted in successful implantation. There were no procedural complications; notably, no cases resulted in stroke, death, pericardial effusion, vascular accidents or device embolization.

The current generation of WM can be successfully implanted using low fluoroscopy and contrast without compromising safety or efficacy using the techniques described.

Keywords Left atrial appendage closure device $\bullet$ Stroke risk reduction $\bullet$ Fluoroscopy • Radiation $\bullet$ X-ray $\bullet$ Contrast

Received: 11.05.18; received in revised form: 25.06.18; accepted: 20.07.18

AF - atrial fibrillation
CVA - cerebral vascular accident
EP - electrophysiology
FT - fluoroscopy time
IAS - inter-atrial septum
ICE - intracardiac echocardiography
LA - left atrium

\section{Abbreviations}

LAA - left atrial appendage

LAO - left atrial appendage occlusion device

OAC - oral anticoagulantion

PA - posterior-anterior

TEE - transesopageal echocardiogram

WM - Watchman left atrial occlusion device 


\section{Introduction}

For patients with $\mathrm{AF}$ who are at elevated risk of thromboembolic CVA, an elevated risk of bleeding and a relative contraindication to the use of anticoagulants, left atrial occlusion device placement is a proven safe and effective alternative to OAC $[1,2]$. WM is a novel technology which involves the delivery of a LAA occlusion device via a transeptal approach and is widely in use in the United States and Europe. While standard implant techniques have been refined since the early clinical trials, device delivery continues to require both fluoroscopy and direct injection of IV contrast into the left atrium to ensure appropriate positioning and to verify appendage occlusion.

\section{Methods}

\section{Patients:}

This is a retrospective observational study and analysis of all 75 consecutive WM implants performed by a single operator at a single center from $12 / 1 / 15$ to 12/31/17. All patients met local implant criteria and provided informed consent. In addition, the majority (99\%) of patients met the more rigorous Medicare implant criteria which included a CHADS $\mathrm{VASC}_{2}$ rank of at least 3 and a HASBLED score of at least [1].

\section{Procedure:}

All patients presented for WM implant at our center's EP or hybrid structural heart laboratory. All patients were diagnosed with well-documented atrial fibrillation and underwent a screening TEE pre-procedure. All patients received general anesthesia including intubation and underwent TEE probe placement at the beginning of the procedure. Intra-procedural TEE was used to rule-out left atrial appendage thrombus and to guide transeptal puncture and WM delivery. Femoral vein access was obtained only under direct ultrasound visualization to avoid vascular accidents. In all patients a figure-of-eight stitch was placed around femoral vein access wires at the beginning of the procedure and were used to achieve hemostasis post-procedure and to minimize the need for manual pressure during recovery. All patients underwent arterial pressure monitoring via radial artery sheath placement. All transeptal punctures were performed under either TEE guidance or, in a minority of patients, using a combination of both TEE and intracardiac echocardiography (ICE) guidance. ICE was performed using the Zonare ultrasound system (Zonare, Mountainview, CA). A single transeptal puncture was performed using the Baylis radiofrequency needle and ProTrack transeptal spiral wire. In those cases where the standard 14 F Boston Scientific transeptal sheath could not be easily passed through the intra-atrial septum (IAS), a $6 \mathrm{~mm} \times 40 \mathrm{~mm}$ angioplasty balloon was inflated across the septum to pre-dilate. All patients presented on chronic warfarin therapy for at least four weeks prior and warfarin was not held prior the procedure. In addition, all patients received heparin bolus and continuous infusion to maintain an activated clotting time (ACT) $>300$ seconds prior to the transeptal puncture and until all catheters and sheaths were removed from the LA. 73 patients (97\%) underwent successful implant with the current generation WM device with sizes ranging from $21 \mathrm{~mm}$ to $33 \mathrm{~mm}$ diameter.

Techniques to reduce fluoroscopy and contrast use

Low fluoroscopy exposure and contrast use were achieved through several measures:

1. Fluoroscopy system adjustment: fluoroscopy exposure can reduced by complying with general principles of radiation protection including maximizing collimating, optimizing projection angle and reducing frame rates. Our standard frame rate is 3.8 frames/ second to start, though this was increased as necessary during contrast injections and device deployment as necessary to optimize imaging. Most implants were performed in a hybrid cardiac catheterization laboratory dedicated to structural heart procedures and equipped with the newest available fluoroscopy software.

2. Saved images: In addition to using a low frame rate, we also used a «single shot» technique where individual one-second exposures were performed and the static image was saved to a separate review screen and used to guide decision-making.

3. Operator preference: perhaps the most critical component to reducing fluoroscopy exposure is operator awareness, motivation and diligence. In our experience, physicians agree to monitor their use and actively «stay off the pedal» when they realize that fluoroscopy is best used in combination with over other forms of imaging (TEE in this case) and not as the sole form of direct visualization.

4. Contrast minimization during appendage cannulation and catheter positioning: we were able to reduce contrast use by administering small (1-2 cc) injections with initial cannulation of the appendage and to confirm distal appendage positioning of the diagnostic pig-tail catheter. We then performed as few longer contrast injections as were necessary to adequately visualize the distal appendage anatomy and os (for sizing and to determine a «landing zone» for the device).

5. Contrast injection during deployment: from the moment deployment is started, small $(1-2 \mathrm{cc})$ injections were performed at short intervals until the device was released.

For this study fluoroscopy and contrast use reduction were seen in the context of an overall «safety first» approach to this procedure. Additional safety steps were taken to reduce the risk of complication including:

- The use of US guidance and micro-puncture kits to access peripheral vessels which minimizes the risk of trauma and effectively eliminates the risk of 
inadvertently puncture of the adjacent artery.

- The use of an RF transeptal needle, atraumatic spiral transeptal wire and (when necessary) a dilating balloon to insure safe and efficient delivery sheath placement.

- Placement of an arterial blood pressure monitoring line to allow for beat-to-beat monitoring of hemodynamics parameters changes and early detection of pericardial effusion.

- The maintenance of an ACT of at least 300 seconds prior to the TSP to minimize the risk of CVA due to LA thromboembolism.

- The use of a figure-of-eight groin closure stitch and an adjustable suture tension device to reducing the risk of hematoma and obviate the need for protamine post procedure.

- Limited transthoracic echocardiogram performed within two to 12 hours post-procedure to document any post-procedure pericardial effusion.

\section{Post implant care:}

All venous sheaths were removed in the EP lab immediately post-procedure irrespective of ACT or INR levels. Hemostasis in the femoral veins was achieved using a «figure of eight» stitch and suture retention device. The arterial monitoring sheath was removed routinely post procedure when the ACT was under 200 seconds. Protamine was not administered. All patients received a post-procedure transthoracic echocardiogram to confirm the absence of pericardial effusion between one and 12 hours after implant. All patients recovered in our post-anesthesia care unit before being transferred to our telemetry unit overnight and were discharged the following day if they met discharge criteria. All patients remained on warfarin and aspirin post-procedure for 6 weeks before switching to aspirin and clopidogrel for an additional 18 weeks, following which all patients were maintained on aspirin only. The 6-week post-implant TEE demonstrated no thrombus or peri-device flow $>6 \mathrm{~mm}$ in diameter and no device embolization in all patients.

\section{Data collection and statistical analysis:}

Statistics are reported for each WM case. Baseline characteristics for all patients are presented. Frequencies and percentages were displayed for categorical variables. Continuous variables were analyzed as median with interquartile range, unless otherwise stated. Descriptive statistics were calculated in total and by procedure year: 2015, 2016, 2017.

\section{Tools}

The primary tools for the statistical analysis were Excel (Microsoft Excel 2016 with the QI Macros 2015 add-in) and RStudio (RStudio v3.4.4 with the ggplot2 package, accessed via RStudio.cloud).

\section{Analysis - Statistical}

The data were cleaned and statistically analyzed using Excel. There was only one observation from 2015 , so, for the purposes of analysis, that observation was removed from the data set during hypothesis testing.

For the descriptive statistics, the median was selected due to several of the variables exhibiting nonnormality. For consistency, median was the preferred method for determining central tendency throughout the analysis. When calculating the interquartile ranges, the Excel function QUARTILE.EXC was employed.

Hypothesis testing used nonparametric approaches. Continuous-variable data were analyzed using the Mann-Whitney U test. For each such variable, the observations were stratified into two groups by year (2016 and 2017). In each case, the null hypothesis was that the two distributions were equal.

Attribute data were analyzed using the 2 Proportions test. For each such variable, the observations were stratified into two groups by year (2016 and 2017). In each case, the null hypothesis was that the difference between the two proportions was zero.

\section{Analysis - Graphical}

Several variables were analyzed graphically using box plots, created in RStudio. In one case (Fluoroscopy Time for 2016), one observation (326 minutes, observation ID 12) was removed. The removal of this outlier did not change the median value (2.8 minutes).

Baseline characteristics:

Between 2015 and 2017, data from 75 procedures were collected retrospectively and presented in Table 1. 73 of 75 patients underwent successful implant. Both patients with failed implant underwent multiple attempts with multiple devices but, due to challenging anatomy and concern for patient safety, the procedures were terminated without successful implant. Patients were a median age of 76 years old and $54.6 \%$ male. Patient characteristics were generally consistent across the duration of the study; no differences reached statistical significance (alpha $=0.05$ throughout this study). The CHADS 2 VASc score remained high (median 4) reflecting multiple risk factors for thromboembolic events. Most patients presented with paroxysmal AF $62.7 \%$ (table 2). Notably, there is no difference in measures of patient size, which can impact radiation absorbed dose, across procedure years (median body surface area $1.99 \mathrm{~m}^{2}$, median body mass index $30.02 \mathrm{~kg} / \mathrm{m}^{2}$ ).

Procedural characteristics are shown in table 3:

1. Procedure time: remained within an expected range at a median of 55 minutes and remained relatively constant.

2. Device size: device sizes ranged from $21 \mathrm{~mm}$ to $33 \mathrm{~mm}$ and the distribution did not change significantly over time.

3. Number of device recaptures: The number of device recaptures reflected the complexity of the cases: the more recaptures (partial or full) reflected more 
Table 1. Clinical characteristics of studied patients

\begin{tabular}{|c|c|c|c|c|c|c|c|}
\hline Attribute & Statistic & $\begin{array}{c}\text { All } \\
\text { observations }\end{array}$ & 2015 & 2016 & 2017 & $\begin{array}{c}\mathbf{p} \\
\text { value }\end{array}$ & Hypothesis Test \\
\hline \multirow{3}{*}{ Age } & count & 75 & 1 & 33 & 41 & & \\
\hline & median & 76 & 63 & 76 & 77 & 0.22 & Mann-Whitney \\
\hline & interquartile range & 9 & $\mathrm{n} / \mathrm{a}$ & 10 & 8 & & \\
\hline Gender & $\%$ who are male & $54.67 \%$ & $100.00 \%$ & $57.58 \%$ & $51.22 \%$ & 0.58 & 2 Proportions \\
\hline \multirow{2}{*}{ Height $(\mathrm{cm})$} & median & 170 & 185 & 170 & 168 & 0.39 & Mann-Whitney \\
\hline & interquartile range & 14.0 & $\mathrm{n} / \mathrm{a}$ & 12.5 & 14.5 & & \\
\hline \multirow{2}{*}{ Weight (kg) } & median & 85.1 & 106.1 & 84.6 & 81.7 & 0.18 & Mann-Whitney \\
\hline & interquartile range & 32.4 & $\mathrm{n} / \mathrm{a}$ & 40.7 & 29.8 & & \\
\hline \multirow{2}{*}{ Last BSA $\left(\mathrm{m}^{2}\right)$} & median & 1.99 & 2.32 & 1.99 & 1.96 & 0.23 & Mann-Whitney \\
\hline & interquartile range & 0.37 & $\mathrm{n} / \mathrm{a}$ & 0.46 & 0.36 & & \\
\hline \multirow{2}{*}{ Last BMI $\left(\mathrm{kg} / \mathrm{m}^{2}\right)$} & median & 30.02 & 31.39 & 29.92 & 30.19 & 0.37 & Mann-Whitney \\
\hline & interquartile range & 10.26 & $\mathrm{n} / \mathrm{a}$ & 12.53 & 7.87 & & \\
\hline \multirow{2}{*}{$\begin{array}{c}\text { CHADS-VASc } \\
\text { Score }\end{array}$} & median & 4 & 2 & 4 & 4 & 0.17 & Mann-Whitney \\
\hline & interquartile range & 2 & $\mathrm{n} / \mathrm{a}$ & 2 & 1 & & \\
\hline $\mathrm{CHF}$ & $\%$ with $\mathrm{CHF}$ & $16.00 \%$ & $0.00 \%$ & $24.24 \%$ & $9.76 \%$ & 0.10 & 2 Proportions \\
\hline \multirow{2}{*}{ LVEF } & median & 60 & 60 & 60 & 60 & 0.23 & Mann-Whitney \\
\hline & interquartile range & 11.0 & $\mathrm{n} / \mathrm{a}$ & 9.5 & 10.0 & & \\
\hline $\begin{array}{c}\text { Obstructive sleep } \\
\text { apnea }\end{array}$ & $\%$ with $O S A$ & $20.00 \%$ & $0.00 \%$ & $15.15 \%$ & $24.39 \%$ & 0.31 & 2 Proportions \\
\hline Diabetes & $\%$ with Diabetes & $38.67 \%$ & $100.00 \%$ & $45.45 \%$ & $31.71 \%$ & 0.22 & 2 Proportions \\
\hline
\end{tabular}

Note: Baseline characteristics. An «n/a» entry means not applicable, since there was only one observation. Where performed, hypothesis testing compares the observations from 2016 with the observations from 2017.

Table 2. Atrial fibrillation type distribution

\begin{tabular}{|c|c|c|c|c|c|}
\hline & & All observations & 2015 & 2016 & 2017 \\
\hline Paroxysmal atrial fibrillation & $\%$ of cases & $62.67 \%$ & $100.00 \%$ & $45.45 \%$ & $75.61 \%$ \\
\hline Persistent atrial fibrillation & $\%$ of cases & $21.33 \%$ & $0.00 \%$ & $36.36 \%$ & $9.76 \%$ \\
\hline Permanent atrial fibrillation & $\%$ of cases & $16.00 \%$ & $0.00 \%$ & $18.18 \%$ & $14.63 \%$ \\
\hline
\end{tabular}

challenging anatomy. $32 \%$ of cases required at least one partial recapture while $24 \%$ of cases required at least one full recapture.

4. Radiation exposure and contrast use: Median fluoroscopy time after the first case in 2015 was low (2.8 minutes) and remained low over time. Median absorbed dose (mGy), a more relevant measure of radiation exposure, was low (median $193 \mathrm{mGy}$ ) and, while it increased over time, the change was not statistically significant.

Complications:

There were no procedural complications.

Procedural success:

Procedural success was defined as successful implant of WMN device which met all PASS criteria: appropriate Position, sufficient Anchor, correct
Size (compression between 8 and 20\%), evidence of adequate Seal (no peri-device leak of $>5 \mathrm{~mm}$ ). Implantation was aborted in $2(2.7 \%)$ patients due to inability to confirm all four PASS criteria after multiple attempts and multiple devices.

\section{Discussion}

As the number and types of invasive cardiac catheterization laboratory procedures continues to grow worldwide each year, so has the concern for the use of procedure-related fluoroscopy. This is due to the understanding that fluoroscopy requires the use of ionizing radiation, that fluoroscopy continues to be the sole or primary imaging mode for such procedures and that patients who are increasingly likely to need multiple radiation-based procedures over their lifetimes. 
Table 3. Procedural characteristics

\begin{tabular}{|c|c|c|c|c|c|c|c|}
\hline Attribute & Statistic & $\begin{array}{c}\text { All } \\
\text { observations }\end{array}$ & 2015 & 2016 & 2017 & $\begin{array}{c}\mathbf{p} \\
\text { value }\end{array}$ & Hypothesis Test \\
\hline Successful implant & $\%$ successful & $97.33 \%$ & $100.00 \%$ & $100.00 \%$ & $95.12 \%$ & 0.15 & 2 Proportions \\
\hline \multirow{2}{*}{ Device size (mm) } & median & 24 & 24 & 24 & 24 & 0.99 & Mann-Whitney \\
\hline & interquartile range & 3 & $\mathrm{n} / \mathrm{a}$ & 3 & 3 & & \\
\hline \multirow{2}{*}{$\begin{array}{l}\text { Preimplant Os size } \\
\qquad(\mathrm{mm})\end{array}$} & median & 20 & 17 & 20 & 21 & 0.49 & Mann-Whitney \\
\hline & interquartile range & 5 & $\mathrm{n} / \mathrm{a}$ & 4 & 5 & & \\
\hline \multirow{2}{*}{$\begin{array}{l}\text { Fluoroscopy } \\
\text { exposure dose } \\
\text { (mGy) }\end{array}$} & median & 193 & 75 & 165 & 225 & 0.15 & Mann-Whitney \\
\hline & interquartile range & 280 & $\mathrm{n} / \mathrm{a}$ & 243 & 285 & & \\
\hline \multirow{2}{*}{$\begin{array}{l}\text { Fluoroscopy time } \\
\text { (min) }\end{array}$} & median & 2.8 & 7.5 & 2.8 & 2.8 & 0.87 & Mann-Whitney \\
\hline & interquartile range & 2.0 & $\mathrm{n} / \mathrm{a}$ & 2.3 & 1.5 & & \\
\hline \multirow{2}{*}{ Contrast volume (cc) } & median & 70 & 65 & 70 & 70 & 0.40 & Mann-Whitney \\
\hline & interquartile range & 50.0 & $\mathrm{n} / \mathrm{a}$ & 58.0 & 50.5 & & \\
\hline \multirow{2}{*}{$\begin{array}{l}\text { Procedure time } \\
\text { (mins) }\end{array}$} & median & 55 & 59 & 55 & 52 & 0.07 & Mann-Whitney \\
\hline & interquartile range & 19.0 & $\mathrm{n} / \mathrm{a}$ & 24.5 & 18.5 & & \\
\hline Partial recaptures & $\%$ of cases & $32.00 \%$ & $100.00 \%$ & $30.30 \%$ & $31.71 \%$ & 0.90 & 2 Proportions \\
\hline Full recaptures & $\%$ of cases & $24.00 \%$ & $0.00 \%$ & $18.18 \%$ & $29.27 \%$ & 0.26 & 2 Proportions \\
\hline
\end{tabular}

Note: Procedural data. An «n/a» entry means not applicable, since there was only one observation. Where performed, hypothesis testing compares the observations from 2016 with the observations from 2017.

In addition, it is well understood that there is no safe level of radiation exposure [3]. The hypothesis regarding the harmful effects of low-dose exposure is extrapolated from the known effects of radiation at high doses, referred to as the «linear non-threshold model» which accepts that risk is related to the cumulative exposure over time. Fortunately, multiple published studies have demonstrated the feasibility, efficacy and safety of minimizing or eliminating fluoroscopy during electrophysiology studies and the techniques which reduce fluoroscopy use appear to be transferable to other interventional procedures, including WM [4-8].

It is well documented that WM is a safe and effective alternative to $\mathrm{OAC}$ in patients with $\mathrm{AF}$ and high risk of CVA and bleeding who also have a contraindication to long-term use of OAC $[1,2]$. While implant is relatively safe, it requires the use of a combination of fluoroscopy and intra-atrial contrast injections under TEE guidance $[9,10]$ with the in associated risks. In spite of attempts to find alternatives to fluoroscopy and contrast use (including reported cases of successful device delivery under ICE guidance), implant techniques have not changed significantly since the device received FDA approval for widespread use in the United States in 2015. Until now there are no published studies that have evaluated a systematic approach to reducing fluoroscopy and contrast use during implant.

Over the course of the first 75 implants at our center by a single operator from December, 2015 to December, 2017 a conscientious effort was made to minimize fluoroscopy and contrast use by employing a variety of techniques and safe practices originally developed for other invasive EP procedures. These data demonstrate that taking such an approach does not increase procedure time beyond what would be expected or result increased complications and does not require additional equipment or operator training beyond what is currently recommended. While this study did not demonstrate a significant reduction in radiation exposure and contrast use over time, median levels started and remained low across time. While median radiation exposure dose did appear to increase over time, this was not statistically significant. Of note, while no direct cause of increased radiation exposure time is obvious from the data, it appears to be due at least in part to the complexity of the cases which appeared to increase in 2017 as we attempted more challenging anatomy. These data reflect a conscientious effort by the operator to ensure patient safety.

\section{Study limitations and future perspective}

This study represents a retrospective observational study by a single operator at a single center; interpretations are limited due to the nature of the data. Additionally, the operator is a relatively highvolume WM implanter who also performs over 100 additional transeptal EP procedures annually and has performed over 500 transeptal procedures in the last 7 years. However, as demonstrated here, achieving these results involved no particular skill set which could not be easily developed by any experienced operator.

This study is also limited by the fact that a comparison 
cannot be made with prior WM data on fluoroscopy and contrast use, as this was not reported in any of the published papers.

Possible future directions include the use of ICE to effectively visualize the LAA, obviating the need for simultaneous TEE. To date, two studies have demonstrated its safety and efficacy in comparison to current implant methods but wide-spread adoption appears to be limited by several factors, including cost $[8,9]$. Finally, the latest generation device, Watchman FLX, holds promise for easier implants due to the limited LAA depth required to deploy the device and greater flexibility in capturing and repositioning.

\section{Conclusion}

This retrospective, observational study demonstrates the safety, feasibility and effectiveness of a comprehensive approach to minimize fluoroscopy and contrast use during WM implants without compromising safety. While neither contrast nor fluoroscopy use decreased over time, both started and remained low. There were no complications associated with this study.

\section{Conflict of interest}

J.A. Reiss declares that there are no conflicts of interest related to this article. D.A. Evans declares that there are no conflicts of interest related to this article.

\section{Funding}

The authors declare that there is no funding related to this article.

\section{Author Information Form}

J.A. Reiss, medical doctor, master of public health, fellow of the American College of Cardiology, fellow of the Heart Rhythm Society, Heart \& Vascular Department, PeaceHealth Southwest Medical Center, 200 NE Mother Joseph Place, Suite 400, Vancouver, WA, United States;

D.A. Evans, Manager, Performance Improvement, PeaceHealth Southwest Medical Center, , 200 NE Mother Joseph Place, Suite 400, Vancouver, WA, United States.

\section{Author Contribution Statement}

$J A R$ - conceptualization, methodology, administration, validation;

$D A E$ - investigation, formal analysis, writing, review.

\section{REFERENCES}

1. Reddy V.Y., Holmes D., Doshi S.K., Neuzil P., Kar S. Safety of percutaneous left atrial appendage closure: results from the Watchman Left Atrial Appendage System for Embolic Protection in Patients with AF (PROTECT AF) clinical trial and the Continued Access Registry.

Circulation. 2011 Feb 1;123(4):417-24. doi: 10.1161/ CIRCULATIONAHA.110.976449.

2. Holmes D.R. Jr., Kar S., Price M.J., Whisenant B., Sievert H., Doshi S.K., Huber K., Reddy V.Y. Prospective randomized evaluation of the Watchman Left Atrial Appendage Closure device in patients with atrial fibrillation versus longterm warfarin therapy: the PREVAIL trial. J Am Coll Cardiol. 2014 Jul 8;64(1):1-12. doi: 10.1016/j.jacc.2014.04.029.

3. Gianni C., Natale A. Reducing radiation exposure in the electrophysiology laboratory: A work in progress. Heart Rhythm. 2017 Jun;14(6):817-818. doi: 10.1016/j.hrthm.2017.02.032.

4. Heidbuchel H., Wittkampf F.H., Vano E., Ernst S., Schilling R., Picano E., Mont L., Jais P., de Bono J., Piorkowski C., Saad E., Femenia F. Practical ways to reduce radiation dose for patients and staff during device implantations and electrophysiological procedures. Europace. 2014 Jul;16(7):94664. doi: 10.1093/europace/eut409

5. Fernández-Gómez J.M., Moriña-Vázquez P., Morales Edel R., Venegas-Gamero J., Barba-Pichardo R., Carranza M.H. Exclusion of fluoroscopy use in catheter ablation procedures: six years of experience at a single center. J Cardiovasc Electrophysiol. 2014 Jun;25(6):638-44. doi: 10.1111/jce.12385

6. Casella M., Pelargonio G., Dello Russo A., Riva S.,
Bartoletti S., Santangeli P., Scarà A., Sanna T., Proietti R., Di Biase L., Gallinghouse G.J., Narducci M.L., Sisto L., Bellocci F., Natale A., Tondo C. "Near-zero" fluoroscopic exposure in supraventricular arrhythmia ablation using the EnSite NavX ${ }^{\mathrm{TM}}$ mapping system: personal experience and review of the literature. J Interv Card Electrophysiol. 2011 Aug;31(2):10918. doi: 10.1007/s10840-011-9553-5

7. Giaccardi M., Del Rosso A., Guarnaccia V., Ballo P., Mascia G., Chiodi L., Colella A. Near-zero x-ray in arrhythmia ablation using a 3-dimensional electroanatomic mapping system: A multicenter experience. Heart Rhythm. 2016 Jan;13(1):150-6. doi: 10.1016/j.hrthm.2015.09.003

8. Yamagata K., Aldhoon B., Kautzner J.. Reduction of Fluoroscopy Time and Radiation Dosage During Catheter Ablation for Atrial Fibrillation. Arrhythmia \& Electropysiology Review 2016;5(2): 144-149. doi: 10.15420/AER.2016.16.2

9. Matsuo Y., Neuzil P., Petru J., Chovanec M., Janotka M., Choudry S., Skoda J., Sediva L., Kurabayashi M., Reddy V.Y. Left Atrial Appendage Closure Under Intracardiac Echocardiographic Guidance: Feasibility and Comparison With Transesophageal Echocardiography. J Am Heart Assoc. 2016 Sep 28;5(10). pii: e003695. doi: 10.1161/JAHA.116.003695.

10. Frangieh A.H., Alibegovic J., Templin C., Gaemperli O., Obeid S., Manka R., Holy E.W., Maier W., Lüscher T.F., Binder R.K. Intracardiac versus transesophageal echocardiography for left atrial appendage occlusion with watchman. Catheter Cardiovasc Interv. 2017 Aug 1;90(2):331-338. doi: 10.1002/ ccd.26805.

To cite: J.A. Reiss, D.A. Evans. Achieving low radiation exposure and contrast usage without compromising safety and efficacy during watchman left atrial appendage occlusion device implant. Complex Issues of Cardiovascular Diseases. 2018; 7 (3): 6-11. DOI: 10.17802/2306-1278-2018-7-3-6-11 